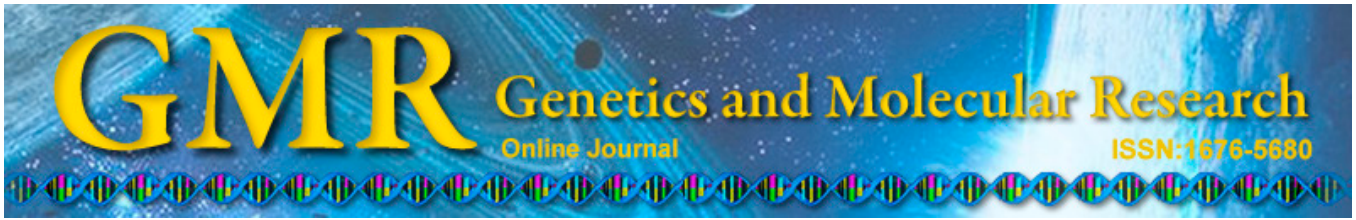

\title{
Molecular characterization of tumor necrosis factor receptor-associated factor 6 (TRAF6) in pearl oyster Pinctada martensii
}

Y. Jian, Q.L. Tian, X.D. Du, Q.H. Wang, R.L. Huang, YW. Deng and S.L. Shi

Fishery College, Guangdong Ocean University, Zhanjiang, China

Corresponding author: X.D. Du

E-mail: gdhddxd@hotmail.com

Genet. Mol. Res. 13 (4): 10545-10555 (2014)

Received November 21, 2013

Accepted February 12, 2014

Published December 12, 2014

DOI http://dx.doi.org/10.4238/2014.December.12.17

\begin{abstract}
Tumor necrosis factor receptor-associated factor 6 (TRAF6) is a key signaling adaptor molecule for tumor necrosis factor receptor superfamily and Toll-like receptor/interleukin-1 receptor family members. It signals the upstream receptors and is involved in a wide range of biological functions, such as immunity and bone metabolism. In this report, the TRAF6 gene from the pearl oyster Pinctada martensii (designated as PmTRAF6) was identified and characterized. The obtained full-length PmTRAF6 cDNA was 2273 bp, containing a 5'-untranslated region (UTR) of $297 \mathrm{bp}$, a 3'-UTR of 128 bp with a 42bp poly (A) tail, and an open reading frame of $1848 \mathrm{bp}$ that encoded 616-amino acid residues. The deduced protein sequence of PmTRAF6 contained a conserved TRAF family motif including a RING-type zinc finger, two TRAF-type zinc fingers, and a coiled-coil region followed by one meprin and TRAF homology domain. Multiple-sequence alignment indicated that TRAF6 was highly conserved among species, and PmTRAF6 showed 53\% sequence identity to Azumapecten farreri and Mizuhopecten yessoensis. Furthermore, an amino acid sequence containing a low-complexity region was inserted in the TRAF6s from mollusk. Quantitative real-time polymerase chain reaction analysis
\end{abstract}


demonstrated that PmTRAF6 was constitutively expressed in all tissues studied, with the most abundant mRNA expression in hepatopancreas and gill in $P$. martensii. After lipopolysaccharide stimulation, the expression of PmTRAF6 mRNA was dramatically upregulated. These results suggested that the obtained PmTRAF6 was a member of the TRAF6 family and perhaps involved in the innate immune response of pearl oyster.

Key words: TRAF6; Cloned gene; Pinctada martensii; Quantitative real-time reverse transcription-polymerase chain reaction

\section{INTRODUCTION}

Tumor necrosis factor receptor-associated factors (TRAFs), which function as adaptor proteins, are signal transducers for the tumor necrosis factor receptor (TNFR) family and Toll-like receptor (TLR)/interleukin-1 receptor (IL-1R) family members (Bradley and Pober, 2001; Wu and Arron, 2003; Kobayashi et al., 2004). Most of the TRAF family members contain a meprin and TRAF homology (MATH) domain in the C-terminal region and RING-type zinc fingers in the $\mathrm{N}$-terminal region followed by a coiled-coil domain. The $\mathrm{C}$-terminal MATH domain is essential for receptor binding. The N-terminal RING-type zinc fingers are crucial for the activation of the downstream signaling cascades (Lomaga et al., 1999; Ye et al., 2002; Wang et al., 2010; He et al., 2006, 2013).

In mammals, there are seven known TRAF family members (TRAF1-7) that play essential roles in signal transduction (Qiu et al., 2009; He et al., 2013). In both the gene structure and protein sequence homology of the C-terminal domain that is responsible for receptor binding, TRAF6 is the most divergent TRAF (Lomaga et al., 1999). With its special C-terminal domain, TRAF6 is the only TRAF that participates in the signaling pathways from the TLR/ IL-1R family (Ye et al., 2002; He et al., 2006). TLR is a member of the pattern recognition receptors and could identify the pathogen-associated molecular patterns of different pathogens (He et al., 2013). IL-1 is crucial in the pro-inflammatory effects through the type-1 IL-1R (He et al., 2006). Although the extracellular domains of TLR and IL-1R are dissimilar, the cytoplasmic regions of each exhibit functional similarity and share a common motif, which is termed the TLR/IL-1R domain (Kobayashi et al., 2004). After activating TLR and IL-1R, a cytoplasmic adapter protein, MyD88, is recruited through their TLR/IL-1R domains, which is followed by the recruitment of IRAK Ser/Thr kinases, and then by association with TRAF6, which mediates the activation of downstream signaling via nuclear factor $\kappa \mathrm{B}(\mathrm{NF}-\mathrm{\kappa B})$ and activator protein 1. With its capacity to signal a broad array of upstream receptors, TRAF6 has a spectrum of biological functions, such as innate and adaptive immunity, stress response, bone metabolism, and the lipopolysaccharide (LPS) signaling pathway (Lomaga et al., 1999). Recently, TRAF6 was reported to be involved in tumor formation and tumor angiogenesis through its function in the activation of NF- $\mathrm{KB}$ and hypoxia-inducible factor-1a (Starczynowski et al., 2011; Sun et al., 2013).

TRAF-6 may be a key molecule that participates in the innate defense system of mollusks (Qiu et al., 2009; He et al., 2013). The pearl oyster Pinctada martensii is the main species for marine pearl production in China and Japan. The identification and characterization 
of TRAF6 in P. martensii will help our understanding of the innate immune response in pearl oyster. Although the TRAF6 gene has been cloned and identified in several marine invertebrates, such as Mizuhopecten yessoensis (He et al., 2013) and Azumapecten farreri (Qiu et al., 2009), the TRAF6 gene has not been characterized in detail and identified in P. martensii. To help our understanding of the innate immune response in pearl oyster, the aim of this research was to obtain the full-length TRAF6 gene and to investigate the TRAF6 gene expression patterns in different tissues and the temporal expression after LPS challenge in P. martensii.

\section{MATERIAL AND METHODS}

\section{Pearl oyster and immune challenge}

Pearl oysters, P. martensii (approximately 2 years of age) were obtained from Liushagang, Zhanjiang, Guangdong Province, China. They were maintained at $25^{\circ}-27^{\circ} \mathrm{C}$ in tanks with recirculating seawater for 1 week before the experiment. In the challenged group, $100 \mu \mathrm{L}$ $10 \mu \mathrm{g} / \mathrm{mL}$ LPS (Sigma, USA) was injected into the adductor muscle of the pearl oysters. In the control group, $100 \mu \mathrm{L}$ phosphate-buffered saline was injected in the same way. Hemocytes were collected from at least six pearl oysters $0,2,4,8,12,24$, and $36 \mathrm{~h}$ after injection, immersed in Trizol (Invitrogen, USA), and stored in liquid nitrogen.

\section{Rapid amplification of cDNA ends (RACE)}

5'/3'-RACE was applied to obtain the full-length cDNA of PmTRAF6 using the SMART RACE cDNA amplification kit (Clontech, Japan). Total RNA for the RACE reaction was extracted from the hemocytes of $P$. martensii using Trizol reagent. Gene-specific primers were designed based on the nucleotide sequences of the PmTRAF6 cDNA fragment obtained from the transcriptome of $P$. martensii (Zhao et al., 2012). Table 1 shows the polymerase chain reaction $(\mathrm{PCR})$ primers used in this study.

Table1. Primers for gene amplification and characterization.

\begin{tabular}{lll}
\hline Primer & Sequence & Application \\
\hline$\beta$-actin-F & 5'-TCCCTGGAGAAGAGC-3' & qRT-PCR \\
$\beta$-actin-R & 5'-AGTTCGTGGATGCC-3' & qRT-PCR \\
3'RACE-PmTRAF1 & 5'-ACGGGCATGTAATGGCTTTTTTGTTTGG-3' \\
3'RACE- PmTRAF 2 & 5'-TCACAGTCGTGTGGTCGATGACCAACGA-3' & Inner PCR \\
5'RACE- PmTRAF 1 & 5'-ATCCCTCATCACGACTTTGGTGTCCGAA-3' & Outer PCR \\
5'RACE- PmTRAF 2 & 5'-CAACATACTCTCCGACAACACGGCGTTA-3' & Inner PCR \\
PmTRAF-F & 5'-GATGGAAACGCTTGTAGCGA-3' & Outer PCR \\
PmTRAF-R & 5'-AGCACAGTCAAAGGGAGGAA-3' & qRT-PCR \\
\hline
\end{tabular}

\section{DNA sequencing and bioinformatic analysis}

The PCR products of the 5'- and 3'-ends were extracted, cloned into the pGEM-T easy vector (Promega), and sequenced. All the obtained sequences were analyzed using the Basic Local Alignment Search Tool (BLAST) available at the National Center for Biotechnology 
Information (NCBI; http://www.ncbi.nlm.nih.gov/). The open reading frame (ORF) was obtained using the ORF Finder tool (http://www.ncbi.nlm.nih.gov/gorf/orfig.cgi). The molecular weight and theoretical isoelectric point were analyzed using a ProtParam tool (http://web. expasy.org/cgi-bin/protparam/protparam). The MEGA 4.0 software was used to construct the phylogenetic tree.

\section{Tissue-specific expression and temporal expression of PmTRAF6 after LPS stimulation}

The adductor muscle, gill, mantle, hepatopancreas, gonad, and hemocytes were collected and immediately stored in liquid nitrogen. Total RNA was isolated using Trizol reagent according to manufacturer instructions. RNA integrity was analyzed by electrophoresis on $1.2 \%$ agarose gels. The RNA concentration was examined using a NanoDrop ND1000 spectrophotometer. The total RNA was reverse transcribed with oligo (dT)16 and M-MLV reverse transcriptase (Clontech). PmTRAF6 mRNA expression was determined by quantitative real-time reverse transcription-PCR (qRT-PCR) with $\beta$-actin as an internal control (Jiao et al., 2012). Amplifications were conducted using SYBR green according to the manufacturer protocol. The qRT-PCR assay was performed in a $\mathrm{C} 1000^{\mathrm{TM}}$ thermal cycler $\mathrm{CFX} 96^{\mathrm{TM}}$ real-time system (Bio-Rad, USA).

\section{Statistical analysis}

The data from the experiments were analyzed using SPSS 13.0. P values less than 0.05 were considered to be statistically significant.

\section{RESULTS}

\section{cDNA cloning, sequencing, and characterization of the PmTRAF6 gene}

Based on the unigene sequence, which was annotated as TRAF6 in the transcriptome database of $P$. martensii, four PmTRAF6 gene-specific primers were designed to amplify the 5'- and 3'-nucleotide sequences by 3'- and 5'-RACE technology. The PCR products were cloned and sequenced. Figure 1 shows that the complete sequence of the PmTRAF6 cDNA was $2273 \mathrm{bp}$, containing a 5'-untranslated region (UTR) of $297 \mathrm{bp}$, an ORF of $1848 \mathrm{bp}$, and a 3'-UTR of 128 bp with a 42-bp poly (A) tail.

\section{Characterization of the PmTRAF6 deduced amino acid sequence}

The deduced protein sequence of PmTRAF6 was 616 amino acids with a mature molecular weight of $70.2 \mathrm{kDa}$ and a theoretical isoelectric point of 6.42 . The deduced amino acid sequence of PmTRAF6 contained characteristic TRAF family motif predicted by the SMART program. One RING-type zinc finger was located between amino acids 60 and 98 . There were two TRAF-type zinc fingers, which were found at amino acids 143-195 and 197-244. One coiled-coil region was located between amino acids 428 and 458, followed by one MATH domain that was predicted between amino acids 468 and 595 (Figure 1). 


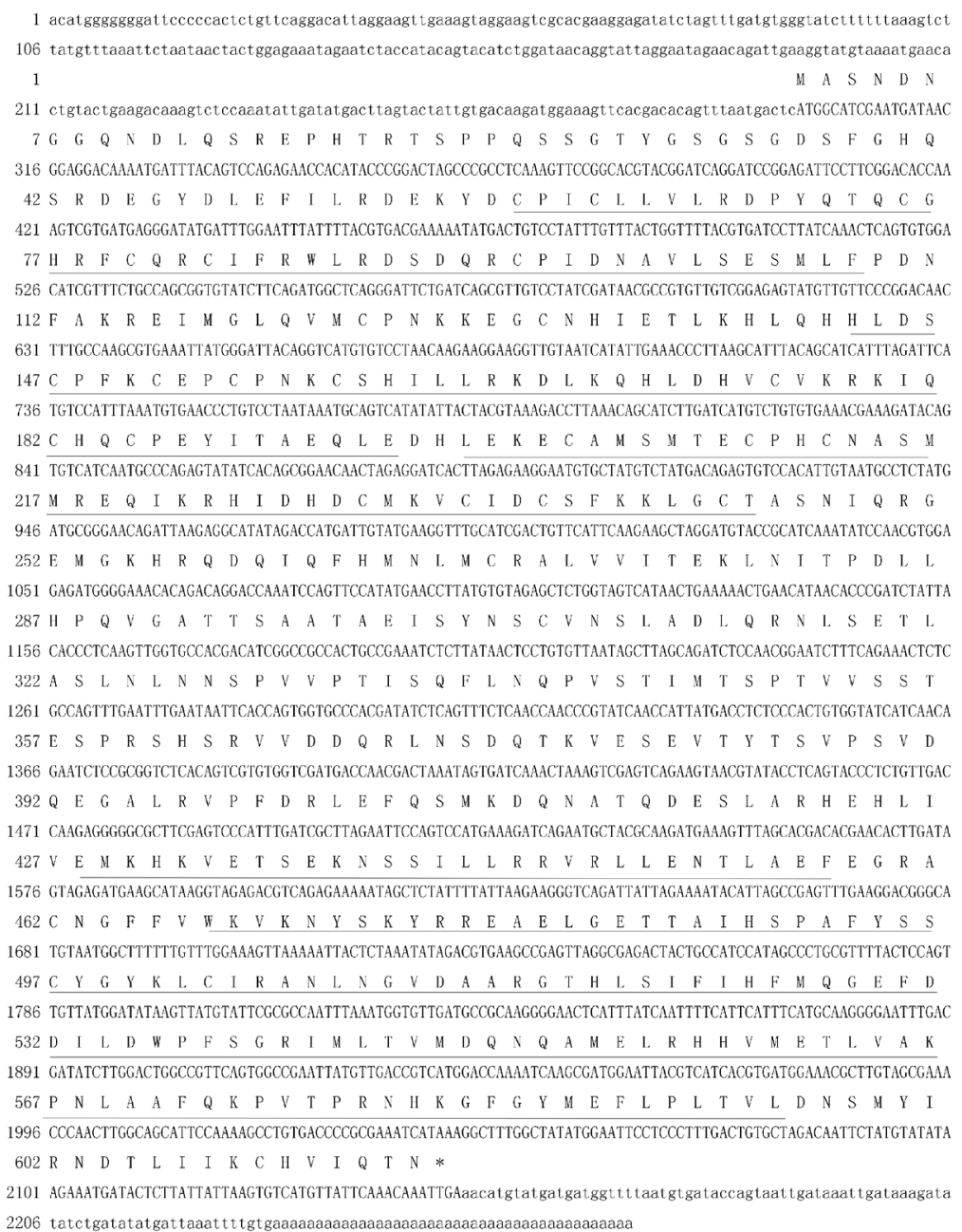

Figure 1. Nucleotide and amino acid sequence of PmTRAF6. Numbers on the left indicate positions of the nucleotides in the PmTRAF6 cDNA sequence and the amino acid residues in the deduced protein. The sequence motif RING-type zinc finger (amino acids 60-98), two TRAF-type zinc fingers (amino acids 143-195 and 197-244), coiled-coil region (amino acids 428-458), and the MATH domain (amino acids 468-595) are underlined.

\section{Homology analysis of PmTRAF6}

The NCBI protein BLAST program was used to analyze the homology of the deduced amino acid sequence of PmTRAF6. PmTRAF6 had significant homology to a variety of TRAF6s that were previously submitted to the database. The sequence of PmTRAF6 showed $53 \%$ sequence identity to the TRAF6 proteins of $A$. farreri and $M$. yessoensis, $47 \%$ identity to that of Euprymna scolopes, and 32\% identity to that of Strongylocentrotus purpuratus. Multiple-sequence alignment with other known TRAF6 proteins showed that most of the identities were located in the characteristic TRAF6 domains (Figure 2). These highly conserved domains indicated that the function of PmTRAF6 was similar to that of other identified TRAF6s. 
Interestingly, similar to A. farreri (Qiu et al., 2009), additional amino acids were located between the second TRAF domain and the coiled-coil region was inserted in P. martensii, $M$. yessoensis, E. scolopes, and S. purpuratus.
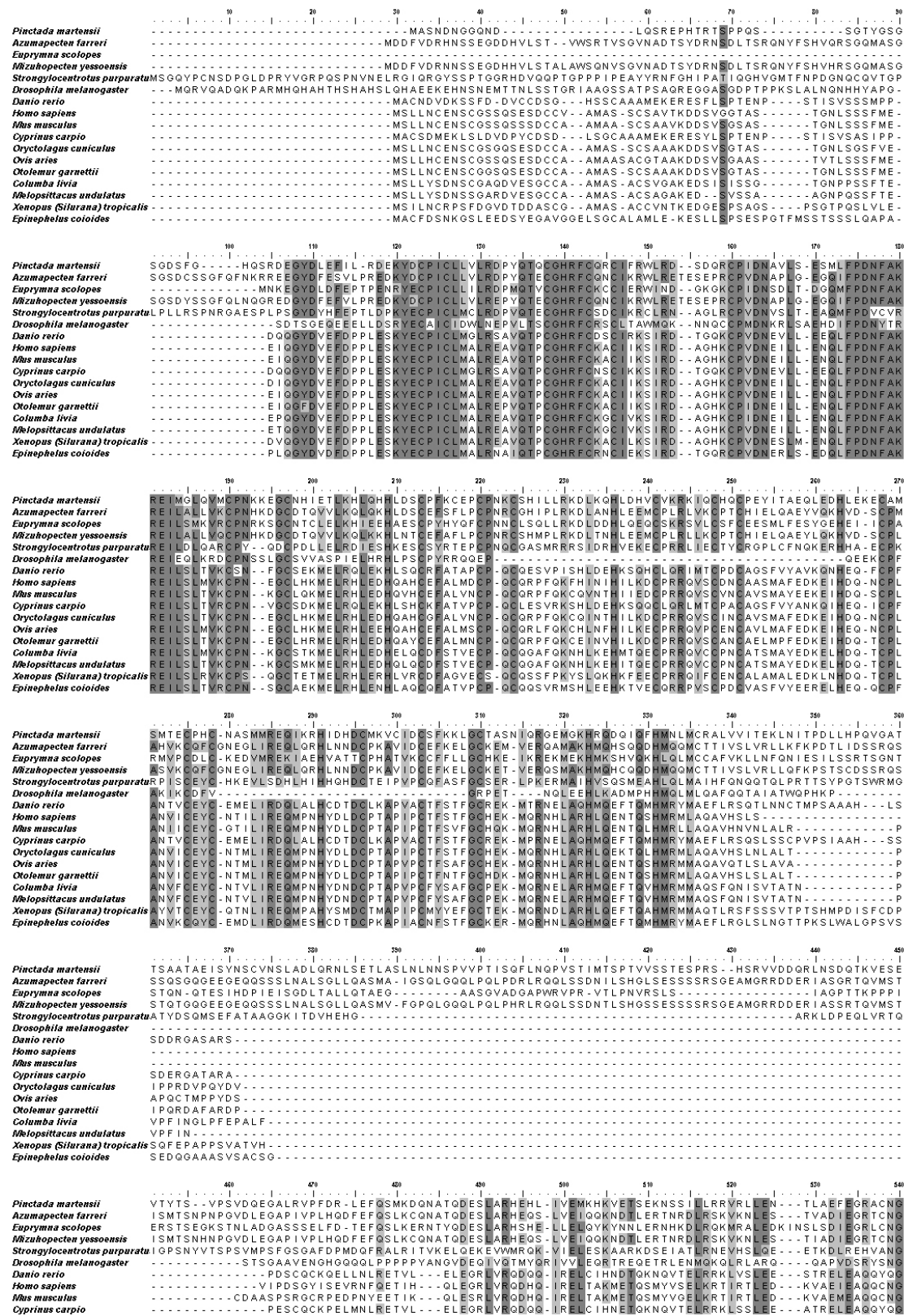

Figure 2. Alignment of the entire amino acid sequence of PmTRAF6 with other known TRAF6 sequences. Identical amino acids are highlighted in black and similar amino acids are highlighted in gray. The origin for each TRAF6 sequence is indicated on the left. 
Further analysis of this insertion using the SMART software indicated that a low-complexity region (LCR) was contained in the TRAF6 proteins of mollusk, including $P$. martensii, A. farreri, and M. yessoensis (Figure 3).

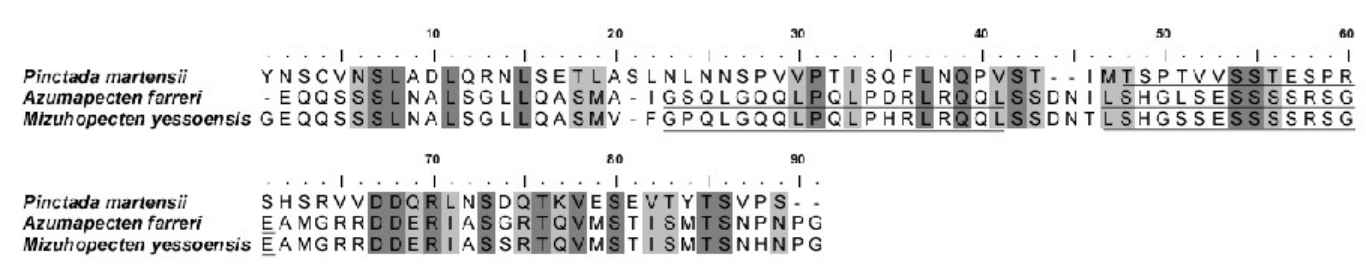

Figure 3. Analysis of the inserted amino acid sequence. The region containing the inserted sequence was analyzed using the SMART tool. The underlined sequences were predicted low-complexity regions.

In order to analyze the phylogenetic relationship between PmTRAF6 and other TRAF6s, a phylogenetic tree was constructed using the MEGA 5.0 software. The results indicated that P. martensii was clustered with TRAF6s from A. farreri and M. yessoensis (Figure 4).

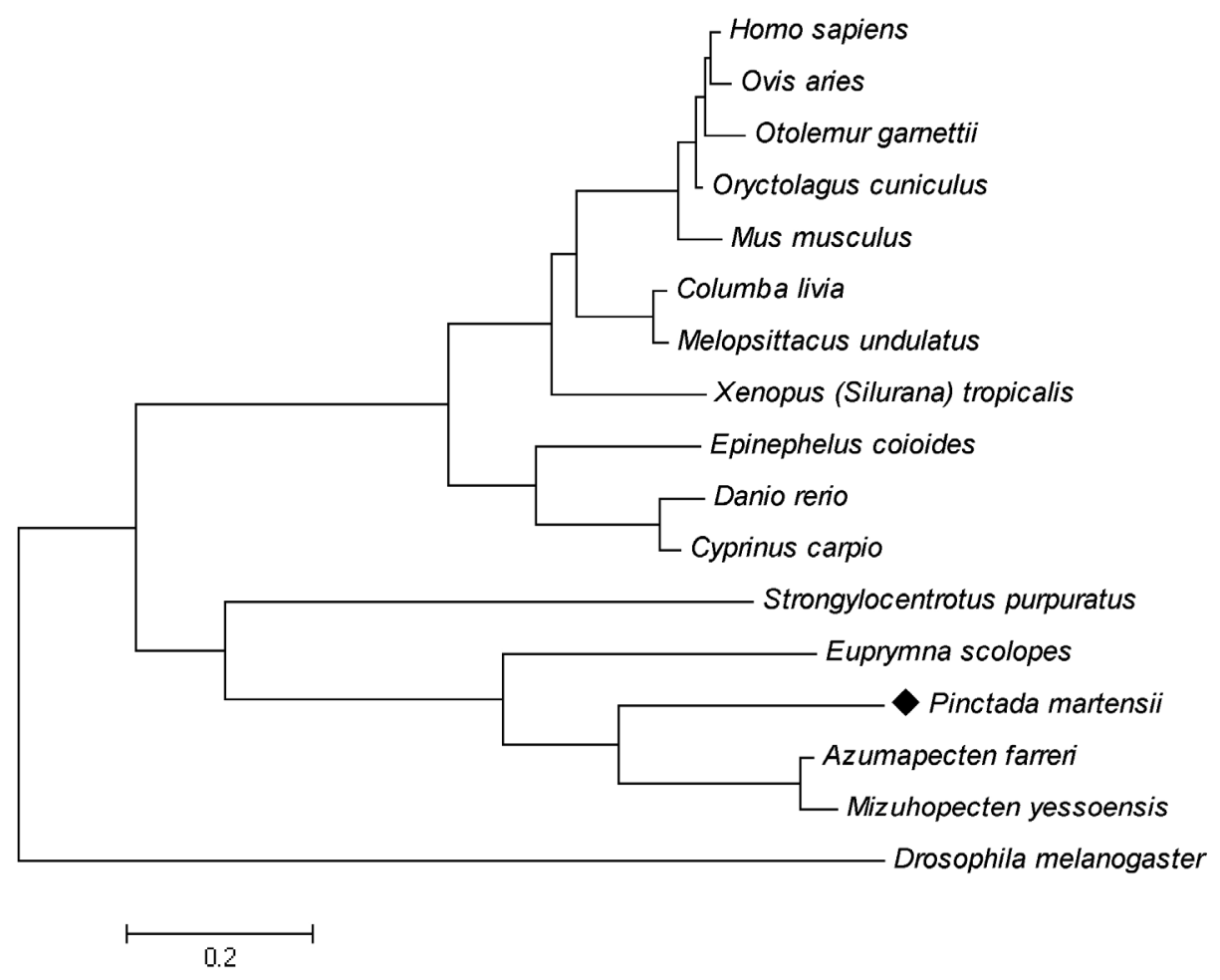

Figure 4. Phylogenic tree showing the relationship between PmTRAF6 and TRAF6s from other species. Phylogenic analysis was performed using the maximum likelihood method in MEGA 5.0 based on the sequence alignment results. 


\section{Quantitative analysis of PmTRAF6 gene expression in different tissues}

Next, qRT-PCR analysis was performed to determine the tissue-specific expression of PmTRAF6 mRNA using $\beta$-actin expression as an internal control. PmTRAF6 mRNA was constitutively expressed in all of the tissues detected (adductor muscle, gill, mantle, hepatopancreas, gonad, and hemocytes). The highest expression was observed in the hepatopancreas and gill, while a relatively lower expression level was detected in the hemocytes (Figure 5).

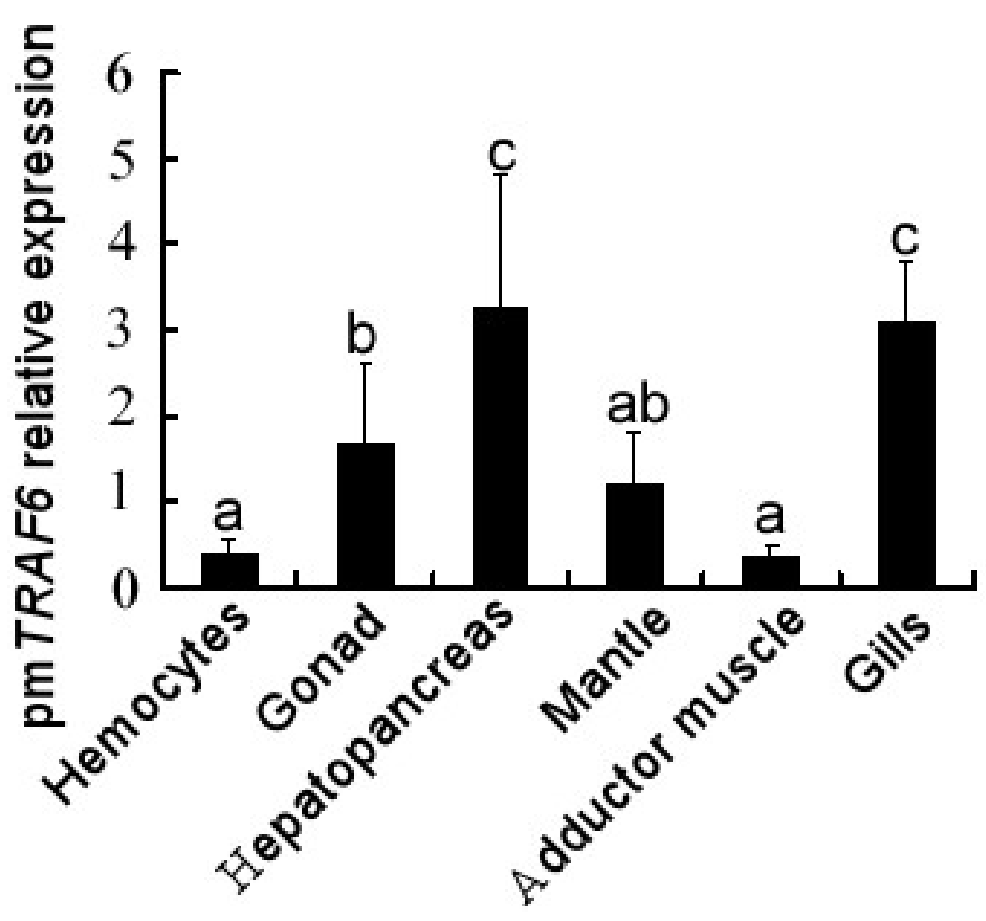

Figure 5. Expression patterns of PmTRAF6 mRNA in different tissues. Quantitative real-time reverse transcriptionpolymerase chain reaction (qRT-PCR) was performed with RNA samples from the adductor muscle, gill, mantle, hepatopancreas, gonad, foot, and hemocytes. The pearl oyster $\beta$-actin gene was used as an internal control. Same letters above the bars represent no significant differences at the $\mathrm{P}>0.05$ level. Different letters above the bars represent significant differences at the $\mathrm{P}<0.05$ level.

\section{Temporal expression pattern of PmTRAF6 after LPS stimulation}

To validate the function of PmTRAF6 in the immune response, we analyzed the temporal expression of the PmTRAF6 gene in hemocytes after LPS stimulation by qRT-PCR. The expression level of PmTRAF6 was significantly increased $4 \mathrm{~h}$ after stimulation and was 4.02-fold higher than that of the control. Then, the expression level gradually decreased and returned to an expression level that was similar to that of the control $24 \mathrm{~h}$ after stimulation (Figure 6). 


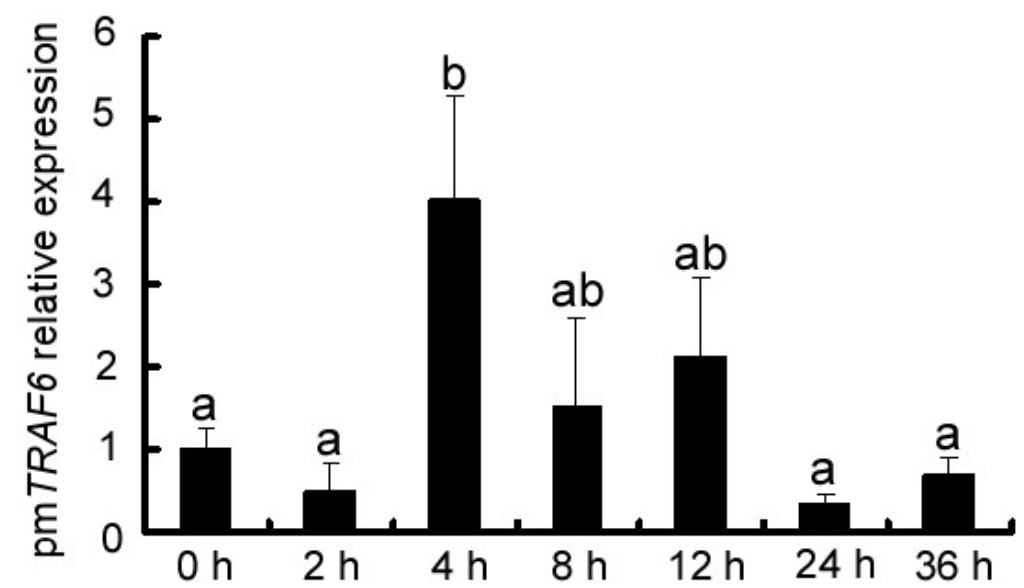

Figure 6. Expression patterns of PmTRAF6 mRNA after lipopolysaccharide (LPS) stimulation. qRT-PCR was performed using the RNA samples from hemocytes $0,2,4,8,12,24$, and $36 \mathrm{~h}$ after LPS stimulation. The pearl oyster $\beta$-actin gene was used as an internal control. Same letters above the bars represent no significant differences at the $\mathrm{P}>0.05$ level. Different letters above the bars represent significant differences at the $\mathrm{P}<0.05$ level.

\section{DISCUSSION}

The IL-1R/TLR superfamily plays critical roles in the innate immunity response to infection and injury (O’Neill, 2002). TRAF has been observed as the major signal transducer for the TNFR and IL-1R/TLR superfamilies. Additionally, numerous biological functions are mediated by TRAF through the induction of cell survival, proliferation, differentiation, and death (Kobayashi et al., 2004, 2009; Lu et al., 2013). TRAF6 is the only TRAF that is crucial for MyD88-dependent signaling pathways originating from the IL-1R/TLR superfamily. Thus, TRAF6 is functionally important in innate immunity. The first experimentally identified molluscan TRAF6 was obtained from A. farreri (CfTRAF6) (Qiu et al., 2009). CfTRAF6 mRNA expression was greatly inhibited by peptidoglycan, indicating its function in the immune response of scallop. Recently, He et al. (2013) obtained the full-length TRAF6 cDNA from M. yessoensis using RACE technology and observed increased TRAF6 mRNA expression after Vibrio anguillarum challenge. TRAF6 was also found in Drosophila melanogaster (DmTRAF6). DmTRAF6 was highly homologous to mammalian TRAF6 and was also identified as a downstream adaptor for TLR (Shen et al., 2001). These reports suggested that TRAF6 was highly conserved and functionally important in the innate immunity of organisms ranging from insect to mammal. To help our understanding of the innate immunity in pearl oyster, we obtained the TRAF6 gene from $P$. martensii and identified its function in the innate immune response.

The full-length PmTRAF6 gene was $2541 \mathrm{bp}$ and encoded 616 amino acids. The deduced protein sequence of PmTRAF6 shared a high degree of identity with other TRAF6s. Meanwhile, PmTRAF6 also contained the three conserved TRAF6 motifs, suggesting functional homology with other TRAF6s. Comparing TRAF6s from vertebrate and fruit fly revealed an insertion in the TRAF6 protein sequences of marine invertebrate, including $P$. martensii, M. yessoensis, E. scolopes, S. purpuratus, and A. farreri. Among these five TRAF6s, only the TRAF6s of mollusk were predicted to contain an LCR. LCRs in a protein are regions of biased composition, normally consisting of a regular repeat, cryptic repeat, and single- 
amino acid repetitions. These LCRs could provide abundant material for new functions (RadóTrilla and Albà, 2012). Additionally, these regions are not strongly conserved in length and evolve rapidly, although many participate in crucial molecular functions ( $\mathrm{Li}$ and Kahveci, 2006). Some types of LCRs are usually found in proteins with particular functional classes, especially transcription factors and protein kinases (Faux et al., 2005). Compared with proteins without LCRs, proteins containing LCRs tend to have more interactions with other proteins (Faux et al., 2005). We proposed that TRAF6s in mollusk may have the ability to combine with other receptors and mediate other signal transductions, but this requires further experimental validation.

To explore the biological function of PmTRAF6 in P. martensii, tissue expression patterns were investigated using qRT-PCR. PmTRAF6 mRNA was highly expressed in the hepatopancreas and gills. The hepatopancreas of mollusks secretes various enzymes to hydrolyze microorganisms (Tiscar and Mosca, 2004). The molluscan gill is the main interface between aquatic organisms and the external environment and is considered as the first line of defense against bacterial infection (Lee et al., 2013). The higher expression of PmTRAF6 in these two organs suggested that it plays a role in the innate immune response.

The hemocytes of mollusk were believed to play crucial roles in the innate immune response. However, a relatively low expression level was observed in hemocytes, which was similar to the expression in hemocytes of $A$. farreri (Qiu et al., 2009). We speculated that the expression of PmTRAF6 in hemocytes may be induced in a stressed state. Then, the temporal expression of PmTRAF6 after LPS challenge in hemocytes was examined to determine if PmTRAF6 was involved in the innate immune response. LPS molecules are large and found in the outer membrane of Gram-negative bacteria. LPS functions as an endotoxin that elicits a strong immune response in animals (Kilár et al., 2013). In mammals, LPS responses are mediated by TLR4 (Chow et al., 1999). As the key signaling adaptor in the TLR signal pathway, TRAF6 could transmit the LPS signal to activate a transcription factor, NF- $\mathrm{BB}$, and other downstream kinases (Wong et al., 2004). In P. martensii, PmTRAF6 increased significantly in the first $4 \mathrm{~h}$ after LPS treatment, suggesting that PmTRAF6 is involved in LPS signaling and thus plays a role in the innate immune response of $P$. martensii.

\section{CONCLUSION}

In conclusion, using the partial sequence obtained from the transcriptome of $P$. martensii, we obtained the full-length PmTRAF6 cDNA and analyzed the characteristics of the ORF and the peptide sequence. The deduced protein sequence of PmTRAF6 contained a conserved TRAF family motif and was highly conserved among species. Furthermore, an LCR was inserted in the molluscan TRAF6s that was not present in the TRAF6 of other species. PmTRAF6 mRNA was highly expressed in the hepatopancreas and gill. After LPS stimulation, the expression of PmTRAF6 mRNA was significantly upregulated, suggesting that PmTRAF6 was involved in the innate immune response of pearl oyster. Further studies on the mechanism that controls the function of PmTRAF6 are necessary.

\section{ACKNOWLEDGMENTS}

Research supported by grants of the National Natural Science Foundation of China (\#31272635, \#41206141, and \#31202023), the Guangdong Natural Science Foundation 
(\#S2012040008042), the Guangdong Province Breeding Project Fund (\#2012LYM_0074), and the Natural Foundation of Guangdong Ocean University (\#1212318).

\section{REFERENCES}

Bradley JR and Pober JS (2001). Tumor necrosis factor receptor-associated factors (TRAFs). Oncogene 20: 6482-6491.

Chow JC, Young DW, Golenbock DT, Christ WJ, et al. (1999). Toll-like receptor-4 mediates lipopolysaccharide-induced signal transduction. J. Biol. Chem. 274: 10689-10692.

Faux NG, Bottomley SP, Lesk AM, Irving JA, et al. (2005). Functional insights from the distribution and role of homopeptide repeat-containing proteins. Genome Res. 15: 537-551.

He CB, Wang Y, Liu WD, Gao XG, et al. (2013). Cloning, promoter analysis and expression of the tumor necrosis factor receptor-associated factor 6 (TRAF6) in Japanese scallop (Mizuhopecten yessoensis). Mol. Biol. Rep. 40: 4769-4779.

He L, Wu X, Siegel R and Lipsky PE (2006). TRAF6 regulates cell fate decisions by inducing caspase 8-dependent apoptosis and the activation of NF-kappaB. J. Biol. Chem. 281: 11235-11249.

Jiao Y, Wang H, Du X, Zhao X, et al. (2012). Dermatopontin, a shell matrix protein gene from pearl oyster Pinctada martensii, participates in nacre formation. Biochem. Biophys. Res. Commun. 425: 679-683.

Kilár A, Dörnyei Á and Kocsis B (2013). Structural characterization of bacterial lipopolysaccharides with mass spectrometry and on- and off-line separation techniques. Mass Spectrom. Rev. 32: 90-117.

Kobayashi T, Walsh MC and Choi Y (2004). The role of TRAF6 in signal transduction and the immune response. Microbes Infect. 6: 1333-1338.

Kobayashi T, Kim TS, Jacob A, Walsh MC, et al. (2009). TRAF6 is required for generation of the B-1a B cell compartment as well as T cell-dependent and -independent humoral immune responses. PLoS One 4: e4736.

Lee Y, Wickamarachchi WD, Whang I, Oh M, et al. (2013). Immune response-related gene expression profile of a novel molluscan IкB protein member from Manila clam (Ruditapes philippinarum). Mol. Biol. Rep. 40: 1519-1527.

Li X and Kahveci T (2006). A novel algorithm for identifying low-complexity regions in a protein sequence. Bioinformatics 22: 2980-2987.

Lomaga MA, Yeh WC, Sarosi I, Duncan GS, et al. (1999). TRAF6 deficiency results in osteopetrosis and defective interleukin-1, CD40, and LPS signaling. Genes Dev. 13: 1015-1024.

Lu Y, Li C, Zhang P, Shao Y, et al. (2013). Two adaptor molecules of MyD88 and TRAF6 in Apostichopus japonicus Toll signaling cascade: molecular cloning and expression analysis. Dev. Comp. Immunol. 41: 498-504.

O'Neill LA (2002). Signal transduction pathways activated by the IL-1 receptor/Toll-like receptor superfamily. Curr. Top. Microbiol. Immunol. 270: 47-61.

Qiu L, Song L, Yu Y, Zhao J, et al. (2009). Identification and expression of TRAF6 (TNF receptor-associated factor 6) gene in Zhikong scallop Chlamys farreri. Fish Shellfish Immunol. 26: 359-367.

Radó-Trilla N and Albà M (2012). Dissecting the role of low-complexity regions in the evolution of vertebrate proteins. BMC Evol. Biol. 12: 155.

Shen B, Liu H, Skolnik EY and Manley JL (2001). Physical and functional interactions between Drosophila TRAF2 and Pelle kinase contribute to Dorsal activation. Proc. Natl. Acad. Sci. U. S. A. 98: 8596-8601.

Starczynowski DT, Lockwood WW, Deléhouzée S, Chari R, et al. (2011). TRAF6 is an amplified oncogene bridging the RAS and NF-кB pathways in human lung cancer. J. Clin. Invest. 121: 4095-4105.

Sun H, Li XB, Meng Y, Fan L, et al. (2013). TRAF6 upregulates expression of HIF-1 $\alpha$ and promotes tumor angiogenesis. Cancer Res. 73: 4950-4959.

Tiscar PG and Mosca F (2004). Defense mechanisms in farmed marine molluscs. Vet. Res. Commun. 28 (Suppl 1): 57-62.

Wang KZ, Galson DL and Auron PE (2010). TRAF6 is autoinhibited by an intramolecular interaction which is counteracted by trans-ubiquitination. J. Cell. Biochem. 110: 763-771.

Wong F, Hull C, Zhande R, Law J, et al. (2004). Lipopolysaccharide initiates a TRAF6-mediated endothelial survival signal. Blood 103: 4520-4526.

Wu H and Arron JR (2003). TRAF6, a molecular bridge spanning adaptive immunity, innate immunity and osteoimmunology. Bioessays 25: 1096-1105.

Ye H, Arron JR, Lamothe B, Cirilli M, et al. (2002). Distinct molecular mechanism for initiating TRAF6 signalling. Nature 418: 443-447.

Zhao X, Wang Q, Jiao Y, Huang R, et al. (2012). Identification of genes potentially related to biomineralization and immunity by transcriptome analysis of pearl sac in pearl oyster Pinctada martensii. Mar. Biotechnol. 14: 730-739. 